\title{
A RARE CASE OF ISOLATED MESENTRIC CASTELMAN'S DISEASE WITH REVIEW OF LITERATURE
}

\author{
R. D. Jaykar, Sachin Jadhav, Rahul Wagh.
}

1. Associate Professor, Department of General surgery Dr V.M.GMC, Sholapur, Maharashtra State, India.

2. Assistant Professor, Department of General surgery Dr V.M.GMC, Sholapur, Maharashtra State, India.

3. Resident, Department of General surgery Dr V.M.GMC, Sholapur, Maharashtra State, India.

\section{CORRESPONDING AUTHOR:}

Dr. Rahul Prakash Wagh,

Shradhaniwas,A/P-pimpalwandi,

Tal-junnar, Dist-Pune, Maharashtra,

Pin-412412

E-mail: rahul_wagh09@yahoo.co.in

\begin{abstract}
We report here a rare case of mesenteric castelman's disease presenting as a lump in abdomen. A 11 year old female child was admitted in surgery ward presenting with intermittent right lower quadrant abdominal pain and lump in lower abdomen. CT scan revealed well defined isoattenuating round solid lesion with smooth margins of approximate size $7.2 \times 6.5 \mathrm{~cm}$ showing moderate homogenous enhancement with few central nonenhancing necrotic areas in it. Exact origin of mass could not be ascertained. Findings might represent GIST, Solid ovarian tumour but definite preoperative diagnosis couldn't be confirmed. Patient underwent exploratory laparotomy and it was a solid mesenteric mass, on microscopic examination confirmed it to be hyaline vascular type of Castelman's disease.
\end{abstract}

KEY WORDS: Castelman's disease-mesentery-hyaline-vascular type

INTRODUCTION: Castleman's disease (giant lymph node hyperplasia angiofollicular lymphoid hyperplasia is a rare lymphoproliferative disease of unknown origin, first described by Castleman in 1954. In the majority of the reported cases, the disorder is located in the mediastinum, whereas the mesenteric involvement is very unusual. The disorder is divided in two histological types: a) the hyaline-vascular type, which accounts for more than $90 \%$ of cases and is usually asymptomatic, and b) the plasma cell type, which is almost always associated with systemic manifestations. Although the former type is the most frequent among the patients suffering from the disease, its variant, called lymphoid subtype, has very rarely been reported in the mediastinum and other extrathoracic sites and only once in the mesentery. We present a case of isolated mesenteric Castleman's disease which presented as a palpable abdominal lump.

CASE REPORT: An 11 year old female child was admitted in surgery ward presenting with intermittent right lower quadrant abdominal pain and lump in mid-lower abdomen. On physical examination there was $7 \mathrm{~cm}$ sized palpable lump in the hypogastrium which was nontender\& mobile in all directions. Initial laboratory investigations were within normal limits .X-ray chest and abdomen showed no pathologic findings. The USG of the abdomen revealed7x6 cm solid hypoechoic mass with vascularity within it, anterior to right kidney. No organomegaly was observed. The CT scan revealed 
well defined iso attenuating round solid lesion with smooth margins of approximate size $7.2 \times 6.5 \mathrm{~cm}$ showing moderate homogenous enhancement with few central nonenhancing necrotic areas in it (Fig 1). Exact origin of mass could not be ascertained. Differential diagnosis of GIST, or solid ovarian tumour was made. On exploratory laparotomy, there was evidence of an egg shaped mass located at root of small bowel mesentery with enlarged regional lymph node and it measured $7.3 \times 6.8 \times 6 \mathrm{~cm}$ in size. It was firm to hard and surrounded by thin fibrous capsule (Fig 2).Segment of mesentery that contained the mass with enlarged lymph node was resected along with nearby $20 \mathrm{~cm}$ segment of small intestine and end to end anastomosis was done. Cut surface was homogenous, brown and firm. Histopathological examination was suggestive of atrophic lymphoid follicles surrounded by concentric small lymphocytes with hyalinized central vessels (Fig 4).The interfollicular areas show proliferation of small vessels surrounded by cuff of collagen suggestive of Castelman's disease hyaline vascular type.

DISCUSSION: Castleman's disease is a rare pathologic process, with undefined precise incidence and unknown etiology, characterized by lymph node hyperplasia. Although it is most commonly seen in adults, it can also occur during childhood. Castleman disease is most often found in the mediastinum $(67 \%)$ but has been identified in multiple anatomic locations, including the neck (14\%), the pelvis (4\%), and the axilla (2\%).Two major histologic forms of Castleman disease, the hyaline-vascular and plasma cell types, and, uncommonly, a transitional or mixed form may be seen. The hyaline-vascular type of Castleman disease is found in $90 \%$ of affected patients and $70 \%$ of cases are diagnosed in patients younger than 30 years old. Patients with the hyaline-vascular type are usually asymptomatic; only $3 \%$ of patients present with systemic complaints. The hyaline vascular type tends to be localized, but in rare cases it is multicentric. The plasma cell type is slightly more likely to be multicentric, but it is sometimes localized. Histologically, the hyaline-vascular type shows hypervascular hyaline germinal centers. The follicle center cells are concentrically layered and flattened, with extensive capillary proliferation between follicles and complete effacement of lymphoid sinuses. .The localized hyaline vascular form has a unique indolent lymph node hyperplasia; which can be found in the abdomen, retroperitoneum or any lymph node basin; as a solitary mass. Compared with patients with the hyaline vascular type of Castleman disease, $50 \%$ of patients with the plasma cell type are symptomatic at presentation. Systemic signs and symptoms may include fever, elevated erythrocyte sedimentation rate, anemia, hypergammaglobulinemia, and splenomegaly. Pediatric patients may present with growth disturbance or failure to thrive. The plasma cell type histologically shows sheets of mature plasma cells with normal- to large-size follicular centers. Effacement of lymphoid sinuses is rare. The histology of Plasma cell type is not specific of systemic Castelman's disease and can be found in autoimmune disease, AIDS and in lymph nodes draining carcinoma, so it is imperious to exclude this condition before diagnosing Castelman's disease of PC type. This implies that serologic testing for HIV should be performed whenever a diagnosis of CD is contemplated. The etiology of Castelman's Disease is related to chronic Human Herpes virus 8 (HSV8) ,as HSV8 has been found in lymphoid cells in case of systemic form, or PC type, of Castleman's disease. Castleman disease in the abdomen and pelvis is most commonly described in the radiology literature as a focal enhancing mass of varying locations, including the retroperitoneum, mesentery, portahepatis, and pancreas. The preoperative diagnosis of the disease is still very difficult even with the newest medical imaging. 
The hyaline-vascular type is described as having three patterns on CT: a solitary noninvasive mass; a dominant infiltrative mass with associated lymphadenopathy; or matted lymphadenopathy without a dominant mass. Treatment of Castelman's disease differs between localized and multifocal or systemic forms. The standard therapy of localized form is surgical excision which is curative when resection is complete and en-bloc. No recurrences have been reported after total excision in the literature.

REVIEW OF LITERATURE: We have reviewed the following articles on castelmans disease

J Gastrointestin Liver Dis June2006 Vol.15 N02,171-174- A rare form of isolated mesenteric castelman's disease presenting as an abdominal mass(Isolated mesenteric castelman's disease).The have reported a case of a 22-year old woman with isolated mesenteric Castleman's disease of the lymphoid variant, which presented as a palpable abdominal mass. The final diagnosis was reached after exploratory laparotomy and resection of the tumor. The described case is the first reported case of Castleman's disease of the lymphoid subtype, located in the mesentery. Castleman's disease is a rare disorder characterized by proliferation of the lymphoid tissue. The most frequent location of the disease is the mediastinum. The location of the disease in the mesentery is rare and it is usually associated with the generalized form of the disease.

Yonesei Medical Journal Vol 46, No2, pp.289-291, 2005 -Mesentric Castelman's Disease. Reported a rare case of mesenteric Castleman'sdisease presenting as a mesenteric mass. A 13-yearold female child was admitted to our hospital complaining of intermittent vague abdominal pain. She had hypochromic anemia, thrombocytosis and an elevated erythrocyte sedimentation rate (ESR). Ultrasonography and computed tomography indicated an intraabdominal mass might represent a lymphoma or gastrointestinal stromal tumor or leiomyoma, but the definitive preoperative diagnosis couldn't be confirmed. The surgical resection of the mass revealed the mesenteric hyaline vasculartype Castleman's disease.

World Journal Of Surgical Oncology 2005, 3.33-Surgical management of abdominal and retroperitoneal castelman's disease.

The authors report two cases of localized Castleman's disease in the retroperitoneal space and review the current and recent progress in the knowledge of this atypical disease. The two patients were young healthy women presenting with a hypervascular peri-renal mass suggestive of malignant tumor. Both have been resected in-toto. One of them had an extensive resection with nephrectomy, while the second had a kidney preserving surgery. Pathological examination revealed localized Castleman's disease and surgical margins were free of disease. Postoperative course was uneventful, and after more than 5-years of follow-up no recurrences have been observed. Abdominal and retroperitoneal Castleman's disease could present either as a localized disease or as a systemic disease. Castleman's disease is a lymphoid hyperplasia related to human Herpes virus type 8, which could have an aggressive behavior, similar to that of malignant lymphoid neoplasm mainly with the systemic type, or a benign one in its localized form. Conclusion was localized Castleman's disease should be considered when facing a solid hypervascular abdominal or retroperitoneal mass. A better knowledge of this disorder and its characteristic would help surgeon to avoid unnecessarily extensive resection for this benign disorder when dealing with abdominal or retroperitoneal tumors. Surgical resection is curative for the localized form, when complete, while splenectomy could be indicated for the systemic form. 


\section{CASE REPORT}

American Journal Of Radiology:175, July 2000-CT features of castelman's disease of abdomen and pelvis

Sixteen cases of Castleman disease of the abdomen or pelvis were found. The pathologic diagnosis was established by surgical excision in all patients. Ten women and six men, ranging in age from nine to 79 years (mean age, 39 years), were included. All patients had been examined with contrast-enhanced CT. The hyaline-vascular type was found in 11 patients (69\%), the plasma cell type in three patients (19\%), and a mixed form in two cases $(13 \%)$. Of the 11 patients with the hyalinevascular type, eight (73\%) were symptomatic. The most common symptom was abdominal pain; additional signs and symptoms included weight loss, fatigue, anemia, and elevated erythrocyte sedimentation rate. All three patients with the plasma cell type were symptomatic. Symptoms included fatigue, failure to thrive, fever, anemia, polyclonal hypergammaglobulinemia, elevated erythrocyte sedimentation rate, and splenomegaly. Both patients with the mixed form of the disease were symptomatic, both presenting with abdominal pain and one presenting with anemia. The lesion was an incidental finding in two patients. One patient presented with a palpable abdominal mass. Clinical history was unavailable for one patient. Fifteen patients (94\%) were found to have a single mass on CT: nine in the retroperitoneum or pelvis, three in the portahepatis, and three in the mesentery. One patient presented with generalized lymphadenopathy in the chest, with diffuse abdominal lymph nodes measuring approximately $1 \mathrm{~cm}$. Of the nine cases of focal retroperitoneal or pelvic Castleman disease, seven were located on the left side and two on the right side. Lesions ranged in size from 1 to $18 \mathrm{~cm}$ in maximum diameter (average size, $4.5 \mathrm{~cm}$ ).All tumors had welldefined margins. Tumors less than $5 \mathrm{~cm}$ in diameter generally showed homogeneous enhancement (enhancement defined as increased attenuation relative to the psoas muscle), whereas tumors greater than $5 \mathrm{~cm}$ in diameter generally showed heterogeneous enhancement. Calcification was seen in five (31\%) of 16 patients; punctate calcification was seen in four patients and an "arborizing" or radial pattern of calcification was seen in one patient. Although calcification tended to be seen in larger lesions (average size, $15 \mathrm{~cm}$ ), calcification was also seen in one lesion measuring $3.5 \mathrm{~cm}$.

\section{REFERENCES:}

1. Frizzera G, Massarelli G, Banks PM, Rosai J.A systemic lymphoproliferative disorder with morphologic features of Castleman's disease: pathological findings in 15 patients. Am J SurgPathol 1983;7:211-231

2. Keller AR, Hochholzer L, Castleman B. Hyaline vascular and plasma-cell types of giant lymph node hyperplasia of the mediastinum and other locations. Cancer 1972;29:670-683

3. Bartkowski DP, Ferrigni RG. Castleman's disease: an unusual retroperitoneal mass. J Urol 1988;139:118-120

4. Rahmouni A, Golli M, Mathieu D, Anglade M-C,Charlotte F, Vasile N. Castleman disease mimicking liver tumor: CT and MR features. J Comput Assist Tomogr 1992;16:699-703

5. Garber SJ, Shaw DG. Case report: the ultrasound and computed tomography appearance of mesenteric Castleman disease. Clin Radiol 1991;43:429-430

6. McAdams HP, Rosado-de-Christensen M, FishbackNF, Templeton PA. Castleman disease of the thorax: radiologic features with clinical and histopathologic correlation. Radiology 1998;209:221-228 


\section{CASE REPORT}

7. Wei BP, Taylor R, Chan YF, Waters K, Alex G. Mesenteric Castleman's disease in childhood. ANZ J Surg 2004; 74: 502-504.

8. Larroche C, Cacoub P, Godeau P: La maladie de Castleman. Rev Med Interne 1996, 17:10031013.

9. Seco JL, Velasco F, Manuel JS, Serrano SR, Tomas L, Velasco A: Retroperitoneal Castleman's disease. Surgery 1992, 112:850-855.

10. Shahidi H, Myers JL, Kvale PA: Castleman's disease. Mayo ClinProc1995, 70:969-977.

11. Cesarman E, Knowles DM: The Role of Kaposi's sarcoma-associated herpesvirus (KHSV/HHV-8) in lymphoproliferative diseases. Semin Cancer Biol 1999, 9:165-174.

12. Testa P, Pigne A, Voinnesson A, Vieillefond A, Paillas J: Les hyperplasies lymphoïde sangio folliculaires (maladie de Catsleman) Premiere localisation meso-sigmoïdienne. Chirurgie 1980, 106:156-160.

13. Ebisuno S, Yamauchi T, Fukatani T, Ohkawa T: Retroperitoneal Castleman's disease: A case report and brief review of tumours of the pararenal area. UrolInt 1989, 44:169-172..

14. Takihara H, Yamakawa G, Baba Y, Takahashi M, Ishihara T: Castleman disease unusual retroperitoneal location indistinguishable from malignant tumour in preoperative angiographic appearance. Urology 1993, 41:162-164.

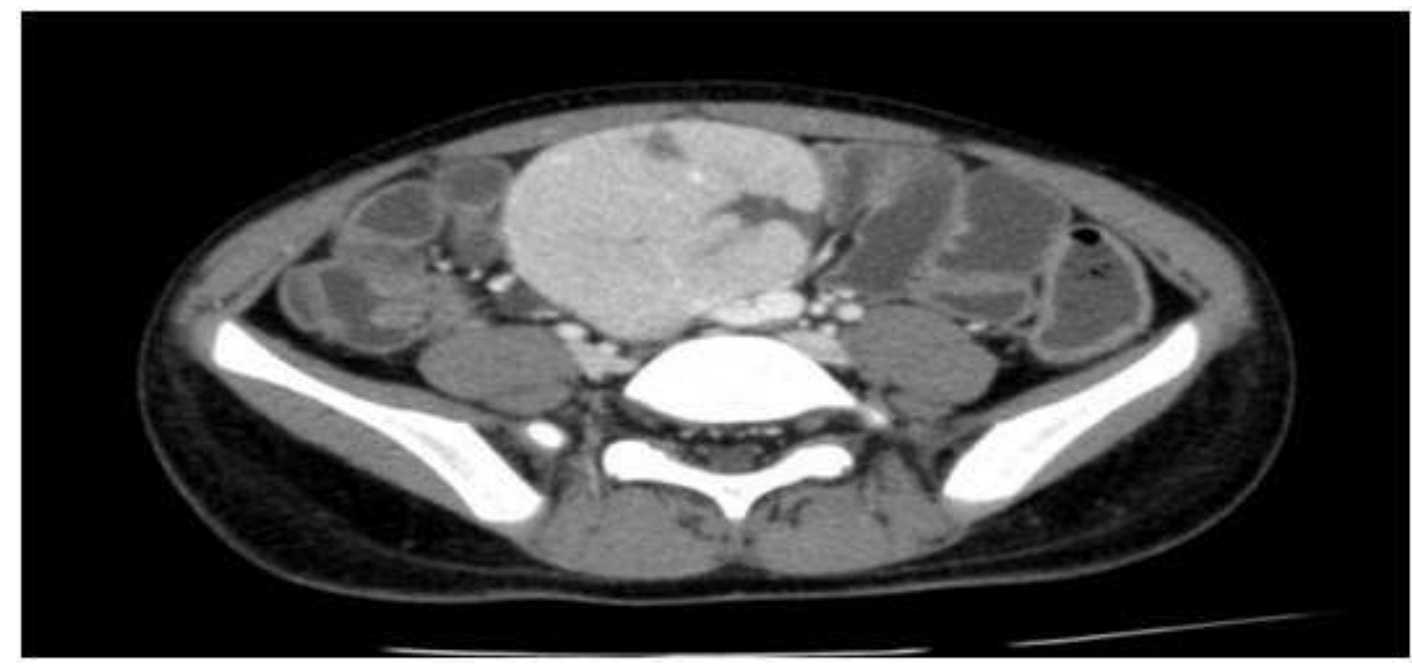

FIG 1: CT scan of the patient shows a well-circumscribed intra-abdominal mass of soft tissue density at the small bowel mesentery. 


\section{CASE REPORT}
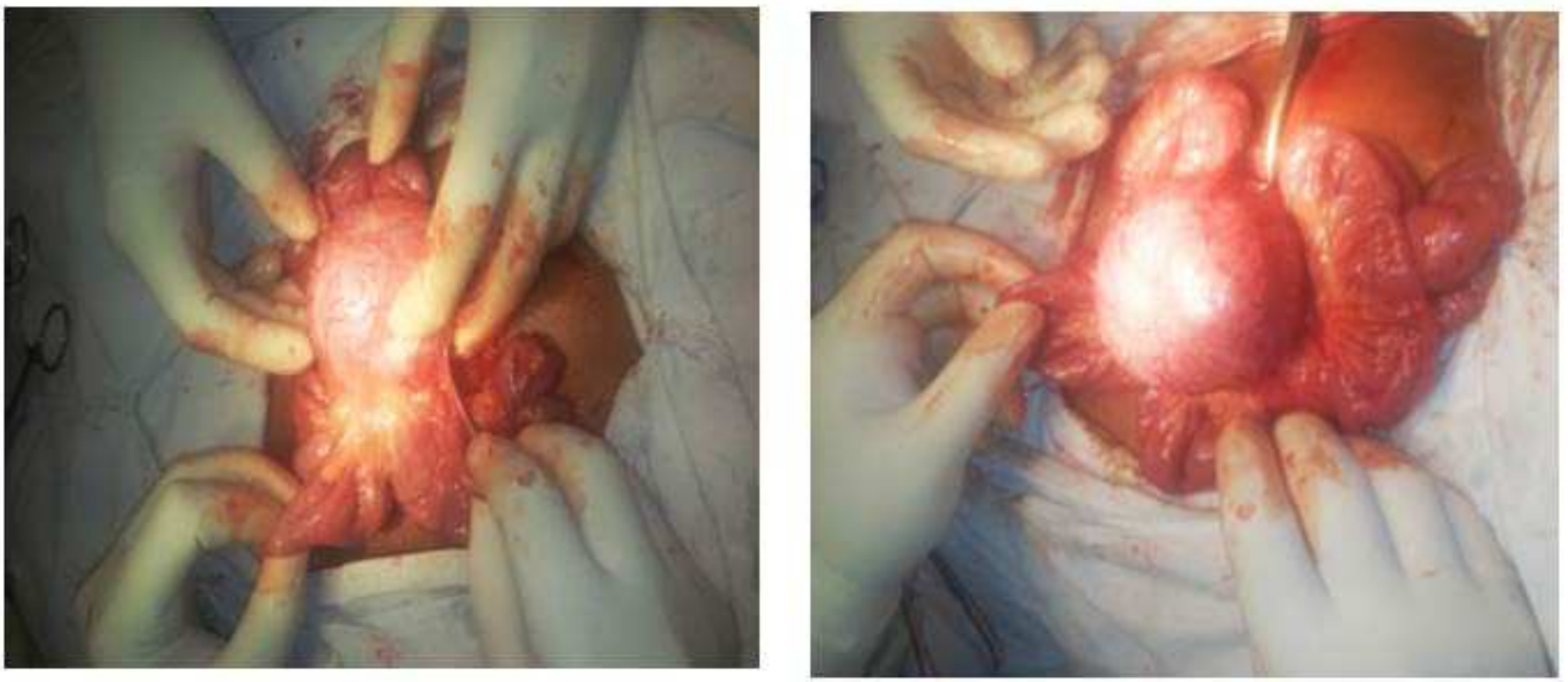

FIG 2: Intraoperative images showing mass arising from base of mesentery
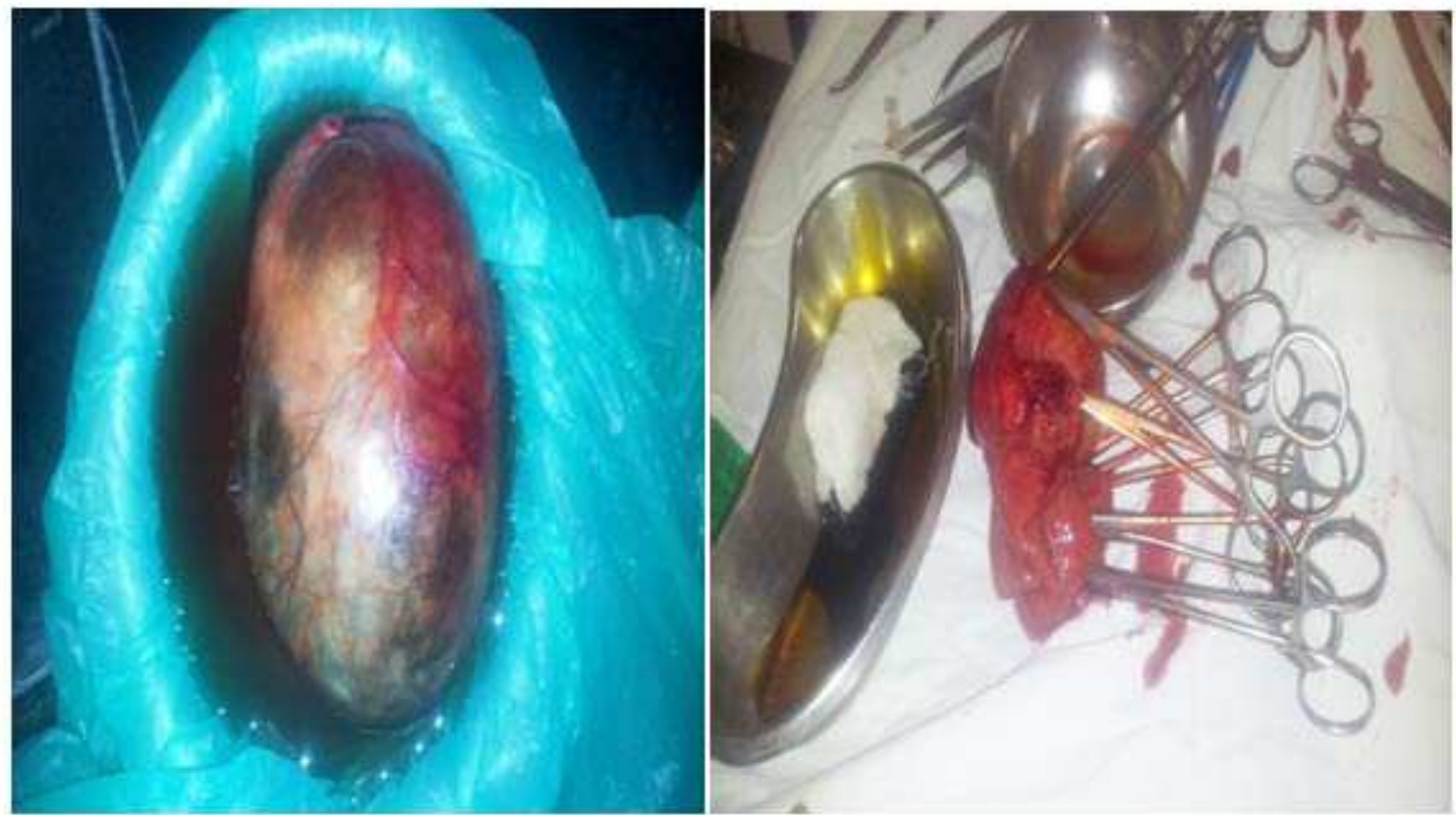

FIG 3: Gross specimen: note the vascularity over the tumour 


\section{CASE REPORT}

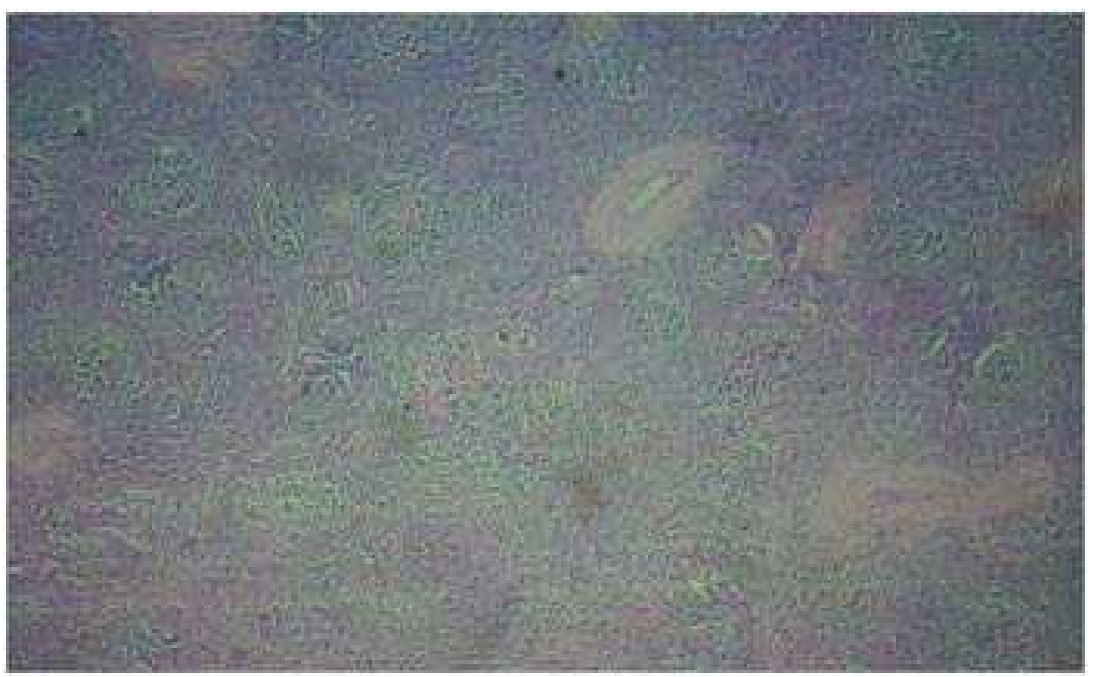

FIG 4: Histological appearance 